\title{
Philosophiques
}

\section{Alain Policar, Ronald Dworkin ou la valeur de l'égalité, Paris, CNRS Éditions, 2015, 223 pages}

\section{Pascal Soligniac}

Volume 43, numéro 1, printemps 2016

URI : https://id.erudit.org/iderudit/1036480ar

DOI : https://doi.org/10.7202/1036480ar

Aller au sommaire du numéro

Éditeur(s)

Société de philosophie du Québec

ISSN

0316-2923 (imprimé)

1492-1391 (numérique)

Découvrir la revue

Citer ce compte rendu

Soligniac, P. (2016). Compte rendu de [Alain Policar, Ronald Dworkin ou la valeur de l'égalité, Paris, CNRS Éditions, 2015, 223 pages]. Philosophiques, 43(1),

174-178. https://doi.org/10.7202/1036480ar d'utilisation que vous pouvez consulter en ligne.

https://apropos.erudit.org/fr/usagers/politique-dutilisation/ 


\title{
Alain Policar, Ronald Dworkin ou la valeur de l'égalité, Paris, CNRS
} Éditions, 2015, 223 pages.

\author{
"Initiation. Cela ne veut pas dire que j'allais parler \\ de petits sujets philosophiques, ni que j'allais donner \\ des éléments simples, afin de préparer l'auditeur à \\ l'activité philosophique. Ni les uns, ni les autres n'existent. \\ Quiconque fait de la philosophie est tout de suite dans \\ les grands sujets et dans la philosophie ${ }^{1}$."
}

Cette remarque de Karl Jaspers au sujet de la philosophie pourrait s'appliquer au livre d'Alain Policar, Ronald Dworkin ou la valeur de l'égalité. Comme le titre l'indique, Policar y présente l'œuvre de Ronald Dworkin, juriste et philosophe politique américain, dont la contribution philosophique reste méconnue à l'extérieur de la sphère anglo-saxonne. Son objectif premier consiste donc à combler cette lacune en déployant un panorama de la philosophie de Dworkin. Tout en accompagnant le lecteur au cœur des enjeux soulevés par le libéralisme dworkinien, l'auteur n'hésite pas à dialoguer avec, et parfois contre les positions soutenues par Dworkin, ce qui confère à son propos une dimension interprétative qui dépasse la seule vulgarisation d'une pensée complexe. Agencé en trois parties, le texte suit l'évolution chronologique des thèmes sur lesquels Dworkin s'est penché: d'abord, la philosophie du droit, ensuite, la philosophie politique, enfin, l'épistémologie et l'ontologie des valeurs.

La première partie retrace la genèse de la théorie du droit dworkinienne, le droit-intégrité, à travers son affrontement avec le positivisme juridique. Policar procède en trois temps. Il caractérise d'abord le positivisme juridique, plus particulièrement la variante développée par Herbert L.A. Hart. Puis, contre cette toile de fond, il fait ressortir la critique de Dworkin à l'endroit de Hart, et enfin explique en substance la théorie du droitintégrité. Cette démarche par contraste, l'auteur éclairant d'abord ce à quoi s'oppose la philosophie dworkinienne, est intéressante à deux titres.

D'une part, le propos du texte gagne en accessibilité: il n'est pas nécessaire de disposer d'une connaissance approfondie des débats qui agitent la philosophie du droit pour saisir l'apport de Dworkin. On le voit, par exemple, dans la présentation de la distinction entre règles de droit et principes juridiques. Si celle-ci constitue un trait incontournable de la pensée de Dworkin, elle n'est intelligible qu'en tant que réponse à la thèse du pouvoir discrétionnaire au juge. Celui-ci l'emploierait pour résoudre les cas qui ne relèvent pas d'une règle de droit explicite ou de précédents. En l'absence de ce point d'opposition et de l'ensemble théorique qui l'entoure, il devient difficile de saisir en

1. Karl Jaspers, Initiation à la méthode philosophique, Paris, Payot, coll. «Petite Bibliothèque Payot ", p. 5 . 
quoi les principes mis de l'avant par Dworkin procèdent d'une conception du juge comme chercheur découvrant la bonne solution à un cas - activité qui relève du registre de la vérité - plutôt que d'une conception du juge comme législateur occasionnel - ce qui relève du registre de l'autorité.

D'autre part, le contraste ainsi créé accentue les traits propres du droit-intégrité dworkinien. Ainsi, Policar souligne fort à propos l'imbrication constante chez Dworkin des méthodes heuristiques et des valeurs substantielles: les principes spécifient à la fois la manière dont le droit doit être compris pour résoudre les cas difficiles mais expriment aussi la valeur au nom de laquelle il importe d'en combler les lacunes. Cette imbrication méthode-valeur devient la trame avec laquelle Policar reconstitue la philosophie du droit de Dworkin. Il analyse donc la valeur qui anime la théorie dworkinienne, l'intégrité. Cette dernière désigne une exigence de cohérence morale selon laquelle une communauté politique doit agir sur une base unique de principes afin de traiter chacun de ses membres en égal. Mais pour ce faire, et c'est la force de l'exposé de Policar que de montrer que la première ne peut se passer de la seconde, il importe d'employer la méthode appropriée, nommément, l'interprétation créative ${ }^{2}$. Celle-ci consiste à interpréter un objet à la lumière des principes qui en font le meilleur exemple en son genre. Deux critères guident le processus interprétatif: l'adéquation de l'interprétation proposée avec la doctrine passée, et la justification de cette dernière au regard des principales valeurs de la morale politique - la justice, l'équité, le traitement de chacun en égal. Sera donc intègre le juge qui emploie l'interprétation créative pour restituer le mouvement allant du cas au droit puis du droit à la morale politique dans son ensemble.

Ce mouvement annonce le déploiement de la pensée dworkinienne à l'ensemble de la philosophie pratique — autant la morale politique que la réflexion sur le bien-vivre. Dans la seconde partie de son livre, Policar regroupe ces thèmes autour de l'étude du libéralisme de Dworkin.

L'auteur identifie immédiatement le noyau foncièrement égalitaire du libéralisme dworkinien. Ce noyau correspond à l'axiome selon lequel les individus sont libres parce que l'État leur doit l'égalité de respect et d'attention. Égalité et liberté s'harmonisent dans la conception qu'a Dworkin de la justice distributive: s'il importe que chacun obtienne sa juste part des ressources matérielles disponibles, cette dernière se mesure en ressources, en fonction des moyens choisis selon les aspirations de chacun, plutôt que du bien-être résultant de la satisfaction des préférences individuelles. Or un tel schème de distribution ne peut opérer sans la garantie des libertés fondamentales puisque leur absence empêcherait l'authentique expression des aspirations individuelles ${ }^{3}$. Policar complète la présentation de l'égalité de

2. Alain Policar, Ronald Dworkin ou la valeur de l'égalité, Paris, CNRS Éditions, 20I 5 , p. 43 .

3. Alain Policar, ibid., p. 8I. 
ressource par une comparaison avec le principe de différence proposé par Rawls et une discussion de l'application de l'égalité de ressource à l'échelle internationale engageant les travaux d'A. Sen et d'A. Renaut ${ }^{4}$.

Ces deux sous-parties du premier chapitre de la deuxième partie représentent un point tournant du livre. L'auteur quitte la posture de présentateur pour se glisser peu à peu dans celle de participant au débat. Cette dimension supplémentaire enrichit significativement le propos du livre qui, de didactique, devient interprétatif. Elle devient plus prégnante à l'intérieur des deux derniers chapitres de la seconde partie.

Policar y explore le contenu que Dworkin attribue à la notion de communauté libérale par le biais de l'argument de l'intégration ${ }^{5}$. Peut-on attribuer un bien-être collectif à une communauté politique fondée sur les libertés individuelles qui ne se réduise pas à la somme de chaque bien-être individuel? Par une démarche qui n'est pas sans rappeler l'harmonisation de l'égalité et de la liberté, Dworkin estime possible d'intégrer bien-être collectif et bien-être individuel à travers une compréhension de la communauté politique comme actant collectif. Si ses actes et ses projets expriment une identité politique commune, elle reste libérale dans la mesure où son existence est tributaire de la coopération concertée d'individus éthiquement autonomes.

Soulignant le rôle charnière joué par la justice entre les deux types de bien-être, Policar aborde le problème de la relation du juste au bien. À la lumière du perfectionnisme ${ }^{6}$, il restitue le débat entre le libéralisme politique de Rawls, chez qui un État juste reste neutre devant les différentes conceptions du bien, et le libéralisme compréhensif de Dworkin, pour qui la justice est un vecteur privilégié du bien-vivre: jusqu'à quel point un libéralisme compréhensif admet-il la promotion d'une conception du bien par les institutions publiques ${ }^{7}$ ? Policar propose ainsi une interprétation du libéralisme de Dworkin qui, si elle ne le place pas fermement du côté des théories perfectionnistes de la justice, du moins y reconnaît des éléments ${ }^{8}$ susceptibles de s'harmoniser avec l'idéal d'autonomie personnelle.

La troisième partie du livre est à l'avenant. Policar y présente l'épistémologie morale qui cimente l'ensemble de l'œuvre de Dworkin. Là, plus encore que dans la partie précédente, Policar interprète la pensée dworki-

4. Alain Policar, ibid., p. 83 et p. 86.

5. Alain Policar, Ronald Dworkin ou la valeur de l'égalité, p. 9I.

6. Le perfectionnisme est une éthique selon laquelle le bien comme la réussite d'une vie peuvent être caractérisés objectivement. Il s'ensuit que l'on peut comparer et classer la réussite de chaque vie indépendamment de la satisfaction qu'en retirent les personnes concernées, et que la justice est conçue comme l'arrangement institutionnel qui engendre le plus de bien objectif (Alain Policar, ibid., p. 204).

7. Alain Policar, ibid., p. Io6.

8. Notamment en ce qui a trait aux politiques culturelles, voir Alain Policar, ibid., p. IIO. 
nienne: «Nous étudierons le système dworkinien en montrant, quelque peu contre son promoteur, qu'il illustre une position réellement métaphysique fondée sur une propédeutique de la vérité ${ }^{9}$. Dit autrement, Policar ne présente Dworkin que pour mieux discuter avec lui - d'où l'intérêt de cette partie qui conclut l'ouvrage.

Elle porte d'abord sur la théorie de la vérité de Dworkin. Il pose comme prémisse de la réflexion axiologique l'indépendance radicale du domaine de la valeur vis-à-vis de celui des faits pour défendre la vérité et l'objectivité des jugements éthiques et politiques. Policar poursuit l'exposé en présentant la critique dworkinienne du scepticisme normatif. Toutefois, cette présentation se mue rapidement en tremplin à partir duquel il envisage l'insertion de l'épistémologie dworkinienne dans un réalisme naturaliste qui en solidifierait l'assise. Ainsi, tandis que Dworkin défend une théorie cohérentiste de la vérité au nom de l'indépendance épistémologique des valeurs, Policar suggère que la thèse de l'intégration des différents registres de la valeur - éthique, morale, et morale politique - serait mieux servie par une conception de la vérité-correspondance ${ }^{10}$. Dans cette optique, les énoncés axiologiques expliqueraient l'expérience morale au même titre que les énoncés scientifiques expliqueraient l'expérience empirique — d'où la référence au naturalisme.

Après un second chapitre consacré à l'indépendance éthique et la liberté politique - nous y reviendrons - Policar poursuit le dialogue avec les thèses les plus récentes de Dworkin au sujet du concept de religion. Celui-ci désigne non pas un culte voué à une entité surnaturelle, mais une "vision du monde aussi profonde que différenciée et complète ${ }^{11}$ ». Policar s'attache ainsi à démontrer le caractère métaphysique d'une réflexion qui, prenant pour point de départ la question de l'ordre de l'univers, convoque l'imbrication du vrai et du beau dans une compréhension générale de la réalité. "Là où il est question d'objectivité, de vérité, de beauté et de cohérence ne sommes-nous pas confrontés à des questions métaphysiques? » demande Policar pour répondre que l'intégration de ces différents champs théoriques dans une optique de connaissance du monde est un projet métaphysique - malgré la méfiance de Dworkin à l'endroit d'un terme qu'il estime trop connoté. Fondée sur une caractérisation de la métaphysique comme "ciment» liant les différentes parties de la réalité à notre esprit, l'interprétation est audacieuse, et l'hypothèse d'une métaphysique dworkinienne qui ne dit pas son nom pose suffisamment de questions pour inviter à la réflexion. Certes, les réponses ne se trouvent

9. Alain Policar, ibid., p. I 6 .

10. Les théories cohérentistes de la vérité attribuent une valeur de vérité à un énoncé selon son insertion cohérente dans un réseau plus large d'énoncés de même nature. Par contraste, les théories de la vérité correspondance font reposer la valeur de vérité d'un énoncé sur son adéquation avec un référent extérieur.

11. Alain Policar, Ronald Dworkin ou la valeur de l'égalité, p. I 53. 
probablement pas dans les écrits de Dworkin; mais c'est là toute la pertinence d'une interprétation réussie: pousser à la recherche.

Le livre de Policar atteint-il son objectif initial, soit faire connaitre l'œuvre de Dworkin? Tout dépend de l'interprétation que l'on en fait.

Si l'on estime que les principes qui animent ce type de monographie sont l'adéquation avec les textes et l'explicitation de la pensée du philosophe concerné telle qu'il la concevait, on émettra quelques réserves au sujet de la composante interprétative inscrite dans Ronald Dworkin ou la valeur de l'égalité. On pourrait aussi regretter que le pragmatisme juridique de R. Posner ne fasse pas l'objet de développements plus consistants: s'il est indéniable que la philosophie du droit de Dworkin plonge ses racines dans la critique du positivisme juridique, le pragmatisme juridique n'a pas moins été un adversaire récurrent du droit-intégrité. De même, on pourrait s'étonner de ne voir la discussion de la conception dworkinienne de la démocratie qu'à la fin de l'ouvrage, en conclusion, plutôt qu'à l'intérieur de la partie consacrée à son libéralisme. Enfin, force est de constater que s'il s'agit bien d'un livre d'introduction à la pensée de Dworkin, ce n'est pas pour autant une œuvre de vulgarisation - quoique cette remarque puisse aussi bien être vue comme un élément positif. En effet, la compréhension des chapitres consacrés à l'épistémologie morale et à la métaphysique suppose une relative familiarité avec la terminologie de ces domaines.

Par contre, si l'on estime que la présentation d'une œuvre est analogue à la démonstration du mouvement — en marchant — en cela qu'elle doit en retracer l'esprit plutôt que la lettre, Policar atteint pleinement son but. Tout en reconnaissant la pertinence des remarques précédentes, on appréciera néanmoins l'aisance avec laquelle Policar établit la portée de la pensée de Dworkin, non seulement en ce qui a trait au spectre des enjeux abordés, mais surtout en quoi celle-ci peut servir de vecteur reliant des questions parfois trop rapidement séparées. Dans cette optique, l'éclairage métaphysique que Policar donne des derniers travaux de Dworkin ouvre des horizons de réflexion autant qu'il explique. Fécond, l'exercice peut aussi susciter des désaccords: d'aucuns pourraient soutenir que le second chapitre de la troisième partie, portant sur l'indépendance éthique et la liberté politique, aurait contribué à présenter une interprétation plus poussée de la relation entre libéralisme dworkinien et perfectionnisme. Cela dit, ce que ce mode d'exposition sacrifie en termes de reproduction objective ou de consensus y gagne en termes de substance: une interprétation créative n'est-elle pas la façon la plus appropriée de présenter l'œuvre d'un philosophe interprétatif?

PASCAL SOLIGNAC

Cégep Marie-Victorin 